\title{
Spatio-Temporal Surface Shear-Stress Variability in Live Plant Canopies and Cube Arrays
}

\author{
Benjamin Walter • Christof Gromke • \\ Katherine C. Leonard · Costantino Manes • \\ Michael Lehning
}

Received: 9 July 2011 / Accepted: 10 December 2011 / Published online: 3 January 2012

(C) Springer Science+Business Media B.V. 2011

\begin{abstract}
This study presents spatiotemporally-resolved measurements of surface shearstress $\tau_{\mathrm{s}}$ in live plant canopies and rigid wooden cube arrays to identify the sheltering capability against sediment erosion of these different roughness elements. Live plants have highly irregular structures that can be extremely flexible and porous resulting in considerable changes to the drag and flow regimes relative to rigid imitations mainly used in other wind-tunnel studies. Mean velocity and kinematic Reynolds stress profiles show that well-developed natural boundary layers were generated above the $8 \mathrm{~m}$ long wind-tunnel test section covered with the roughness elements at four different roughness densities $(\lambda=0,0.017,0.08,0.18)$. Speed-up around the cubes caused higher peak surface shear stress than in experiments with plants at all roughness densities, demonstrating the more effective sheltering ability of the plants. The sheltered areas in the lee of the plants are significantly narrower with higher surface shear stress than those found in the lee of the cubes, and are dependent on the wind speed due to the plants ability to streamline with the flow. This streamlining behaviour results in a decreasing sheltering effect at increasing wind speeds and in lower net turbulence production than in experiments with cubes. Turbulence intensity distributions suggest a suppression of horseshoe vortices in the plant case. Comparison of the surface shear-stress measurements with
\end{abstract}

B. Walter $(\varangle) \cdot$ C. Gromke · K. C. Leonard · M. Lehning

WSL Institute for Snow and Avalanche Research SLF, Flüelastrasse 11, 7260 Davos Dorf, Switzerland e-mail: walter@slf.ch

B. Walter - M. Lehning

CRYOS, School of Architecture, Civil and Environmental Engineering, École Polytechnique Fédéral de Lausanne, Lausanne, Switzerland

K. C. Leonard

Cooperative Institute for Research in Environmental Sciences, University of Colorado at Boulder, 216 UCB, Boulder, CO 80309, USA

C. Manes

Energy and Climate Change Division, School of Engineering and the Environment, University of Southampton, Southampton SO17 1BJ, UK 
sediment erosion patterns shows that the fraction of time a threshold skin friction velocity is exceeded can be used to assess erosion of, and deposition on, that surface.

Keywords Aeolian processes · Drag partitioning · Particle erosion · Surface shear-stress · Turbulent boundary-layer $\cdot$ Vegetation aerodynamics

\section{List of Symbols}

\begin{tabular}{|c|c|}
\hline$A_{\mathrm{f}}$ & Roughness element frontal area \\
\hline$D$ & Average roughness element diameter \\
\hline$Q$ & Mass transport rate \\
\hline$R e_{\mathrm{h}}=U_{\delta} h / v$ & Roughness element Reynolds number \\
\hline$S$ & Ground area per roughness element \\
\hline$U_{\delta}$ & Free-stream velocity \\
\hline$U_{s}$ & Wind speed at Irwin sensor-tube height \\
\hline$c_{1}$ and $c_{2}$ & Constants \\
\hline$d$ & Sand grain diameter \\
\hline$f=20 \mathrm{kHz}$ & Hot-film sampling frequency \\
\hline$f_{\mathrm{c}}$ & Cut-off frequency \\
\hline$h$ & Roughness element height \\
\hline$h_{\mathrm{S}}$ & Irwin sensor tube height \\
\hline$m$ & Parameter relating $\tau_{\mathrm{s}}^{\prime \prime}$ to $\tau_{\mathrm{s}}^{\prime}$ \\
\hline$\Delta p$ & Pressure difference measured by Irwin sensor \\
\hline$\Delta p^{\prime}$ & Fluctuations over pressure signal \\
\hline$u$ & Mean streamwise wind velocity \\
\hline$u^{\prime}$ & Fluctuations in mean streamwise wind velocity \\
\hline$u_{*}=(\tau / \rho)^{1 / 2}$ & Friction velocity \\
\hline$u_{*_{t}}$ & Fluid threshold friction velocity \\
\hline$u_{\tau}=\left(\tau_{\mathrm{s}} / \rho\right)^{1 / 2}$ & Skin friction velocity \\
\hline$u_{\tau t}$ & Fluid threshold skin friction velocity \\
\hline$\overline{u^{\prime} w^{\prime}}$ & Kinematic Reynolds stress \\
\hline$w^{\prime}$ & Fluctuations in mean vertical wind velocity \\
\hline$\beta_{\mathrm{c}}$ & Irwin sensor calibration constant \\
\hline$\beta$ & Ratio of roughness element to surface drag coefficient \\
\hline$\lambda$ & Roughness density \\
\hline$v$ & Kinematic viscosity of air \\
\hline$\psi$ & Percentage of time that threshold skin friction velocity is exceeded \\
\hline$\rho$ & Air density \\
\hline$\sigma$ & Ratio of roughness element basal to frontal area \\
\hline$\sigma_{\mathrm{u}}$ & Standard deviation of wind speed $U_{s}$ \\
\hline$\tau=\rho u_{*}^{2}$ & Total shear stress averaged over whole canopy \\
\hline$\tau_{\mathrm{R}}$ & Shear stress acting on roughness elements \\
\hline$\tau_{\mathrm{s}}(t, x, y)$ & Shear stress acting on surface \\
\hline$\tau_{\mathrm{s}}^{\prime \prime}$ & Spatial peak of temporally-averaged surface shear-stress distribution \\
\hline$\tau_{\mathrm{s} 0}$ & $\begin{array}{l}\text { Spatiotemporally-averaged surface shear stress in the absence } \\
\text { of roughness elements }\end{array}$ \\
\hline$\xi$ & Normalized turbulence intensity \\
\hline
\end{tabular}




\section{Introduction and Background}

Soil erosion, drifting snow, and the entrainment and transport of pollen, seeds and particulate matter are examples of processes governed by airflow over an erodible surface (Bagnold 1943). These processes are directly related to land degradation, desertification, air quality, local water storage in the form of snow, and to reduced biodiversity with strong implications for ecosystems and human societies (Shao 2008). Plants influence these processes by reducing the area of ground exposed to the wind, by trapping particles in motion, by local stress concentration and by absorbing momentum from the flow, resulting in lower surface shear stress $\tau_{\mathrm{s}}$ on the ground beneath the plant canopy (e.g. Wolfe and Nickling 1996).

The distribution of surface shear stress $\tau_{\mathrm{s}}$ is the key to quantifying the sheltering effect of non-erodible roughness elements because its magnitude and spatiotemporal variations determine the onset and degree of differential erosion. The initiation of particle erosion is governed by the fluid threshold friction velocity $u_{*_{t}}$, i.e. particle movement on a surface begins when the friction velocity $u_{*}>u_{*_{t}}$. The threshold value $u_{*_{t}}$ for a given sediment is defined as the friction velocity $u_{*}=\left(-\overline{u^{\prime} w^{\prime}}\right)^{1 / 2}=(\tau / \rho)^{1 / 2}$ in the constant-stress layer at the commencement of particle entrainment. Here, $u^{\prime}$ and $w^{\prime}$ are the fluctuations in the mean streamwise and vertical velocity components, $\tau$ is the total stress on the whole surface and $\rho$ the air density. This threshold value $u_{*_{t}}$ is an important parameter because most mass transport models use $Q \propto\left(u_{*}-u_{*_{t}}\right)^{3}$ to predict the mass transport rate $Q$ (e.g. Anderson and Sorensen 1991).

Numerous investigations of the sheltering effect of plants have been conducted but all of them are constrained by one or more of the following three limitations:

(i) Use of artificial rigid roughness elements (Marshall 1971; Wooding et al. 1973; Gillette and Stockton 1989; Musick et al. 1996; Crawley and Nickling 2003; Brown et al. 2008). Solid rigid cylinders or cubes poorly simulate the aerodynamical shape of live plants. Live plants have highly irregular structures that can be extremely flexible and porous resulting in considerable changes to the drag and flow regimes relative to rigid imitations (Gillies et al. 2002). Crawley and Nickling (2003) conducted drag partition measurements to determine model parameters for the Raupach (1992) drag partition model and found that the surface shear-stress inhomogeneity parameter $m$ should be revised. Brown et al. (2008) found no significant effect of different spatial arrangements of non-erodible roughness elements on the drag partition.

(ii) Field experiments with no control over the wind conditions (Musick and Gillette 1990; Wolfe and Nickling 1996; Wyatt and Nickling 1997; Lancaster and Baas 1998; King et al. 2006; Gillies et al. 2007). In field experiments it is difficult to decouple the influences of the plants on surface shear stress from those induced by the variations in the wind. Wyatt and Nickling (1997) performed shear-stress measurements in sparse desert creosote communities and found greater roughness element drag coefficients for porous shrubs than for solid elements. Gillies et al. (2007) found that the Raupach (1992) drag partition model performed very well for regular arrays of solid elements of different roughness densities within a large open area.

(iii) Spatial and temporal averages: only limited data are available on local peak shearstress values (Crawley and Nickling 2003; King et al. 2006; Brown et al. 2008) or temporal variations of surface shear stress (Sutton and McKenna-Neumann 2008). Sutton and McKenna-Neumann (2008) examined bed level flow patterns around solid cylinders for varying roughness densities and correlated vortices shed by the obstacles with their erosive capability. 
Several wind-tunnel studies have measured the mean surface shear stress on the underlying surface using drag balances to investigate the sheltering effect of non-erodible roughness elements (e.g. Crawley and Nickling 2003). However, Raupach et al. (1993) stated that the peak surface shear stress $\tau_{\mathrm{s}}^{\prime \prime}$ rather than the average surface shear stress on the exposed surface area $\tau_{\mathrm{s}}^{\prime}$ is responsible for the initiation of particle erosion and developed a model that predicts the peak as well as the average surface shear-stress partition:

$$
\left(\frac{\tau_{s}^{\prime \prime}}{\tau}\right)^{\frac{1}{2}}=\left[\frac{1}{(1-m \sigma \lambda)(1+m \beta \lambda)}\right]^{\frac{1}{2}} .
$$

Here, $\lambda=A_{\mathrm{f}} / S$ is the roughness density where $A_{\mathrm{f}}$ is the roughness element frontal area, $S$ is the ground area per roughness element, $\sigma$ is the ratio of roughness element basal to frontal area and $\beta$ is defined as the ratio of the roughness element to surface drag coefficient. The parameter $m$ is defined as $\tau_{\mathrm{s}}^{\prime \prime}=\tau_{\mathrm{s}}^{\prime}(m \lambda)$, which means that the peak surface shear stress is equal to the surface average shear stress at a lower roughness density $(m<1)$. To predict the average surface shear-stress partition $\left(\tau_{\mathrm{s}}^{\prime} / \tau\right)^{1 / 2}$, we set $m=1$.

Some wind-tunnel and field investigations have used Irwin sensors (Irwin 1981; Wu and Stathopoulos 1993) to obtain point measurements of surface shear stress at positions where the largest shear-stress values were expected (Crawley and Nickling 2003; King et al. 2006; Brown et al. 2008). These measurements support the Raupach et al. (1993) model (Eq. 1) very well. To fully quantify the sheltering effect of different roughness elements against erosion, however, the spatial and temporal distribution of surface shear stress $\tau_{\mathrm{s}}(t, x, y)$ on the ground are needed. Sutton and McKenna-Neumann (2008) first measured temporally-resolved surface shear-stress distributions on the surface beneath wooden cylinder arrays using Irwin sensors. Unfortunately it was not possible for them to sample very large arrays that filled the entire floor of the wind tunnel, preventing the generation of a well-developed boundary layer.

Most of the shear-stress ratio measurements referenced above are generally in good agreement with each other. However, variations between them exist, of order $\Delta\left(\tau_{\mathrm{s}}^{\prime} / \tau\right)^{1 / 2} \approx$ $\Delta\left(\tau_{\mathrm{s}}^{\prime \prime} / \tau\right)^{1 / 2} \approx \pm 0.1$. These variations result from different experimental set-ups, types of roughness elements used, and the distribution of the roughness elements over the surface. This makes it difficult for practitioners and modellers to select realistic surface shear-stress values for vegetation canopies over a range of planting densities. It also remains unknown how well rigid and non-porous roughness elements represent the sheltering effect of live plants, and if conclusions drawn from such experiments can be assumed to hold true for live plant canopies in natural environments.

The goal of our wind-tunnel study was to quantify the sheltering capability of different densities of live plant canopies against wind erosion under controlled conditions. The results are compared to similar experiments performed using wooden cube arrays of the same densities because previous wind-tunnel studies quantifying the sheltering effect of plants have mainly used rigid and non-porous plant imitations such as cubes and cylinders for their experiments. Although our live plant arrays are not natural vegetation canopies, the fact that our plants are of similar size, trimmed to a standard height and arranged with regular spacing allows us to systematically investigate the influence of plant flexibility and porosity on the sheltering effect. In addition, the live plant canopies used here are far closer to natural plant canopies than any roughness array used in previous wind-tunnel investigations of shear-stress partitioning. While the use of live plants in wind-tunnel studies is not novel (e.g. Kim et al. 2000 and Burri et al. 2011a,b), this is the first study to investigate the surface shear-stress distribution in live plant canopies of different densities. 


\section{Methods}

We performed wind-tunnel measurements of spatially- and temporally-resolved surface shear-stress distributions $\tau_{\mathrm{s}}(t, x, y)$ on the ground beneath live plant canopies using Irwin sensors (Irwin 1981) (Fig. 1a). For comparison, similar measurements were made substituting rigid cubes at the same locations as the plants (Fig. 1b). Cubes were chosen because of their simple geometry with well-defined flow separation at the edges and because the shearstress distributions in the cube arrays provide a data base for computational fluid dynamics (CFD) model validation. Four different canopy densities $(0,5.25,24.5,55$ roughness elements per $\mathrm{m}^{2}$, hereafter named as the smooth floor, low, medium and high density cases) were investigated at free-stream velocities ranging from $U_{\delta}=8-16 \mathrm{~m} \mathrm{~s}^{-1}$. The corresponding roughness densities $\lambda$ are given in Table 1. Because they streamline with the airflow, the plants frontal area $A_{\mathrm{f}}$ and thus the roughness density $\lambda$ decrease at higher wind speeds (Fig. 1c). To determine $\lambda$, photographs of the plants' upstream vertical faces were taken at the different wind speeds inside the wind tunnel to determine $A_{\mathrm{f}}$ by digital image processing (Fig. 1d). For an overview of the experiments and a summary of the measurement results see Table 1.

The SLF (WSL Institute for Snow and Avalanche Research, Davos, Switzerland) boundary-layer wind tunnel is $18 \mathrm{~m}$ long in total, has an $8 \mathrm{~m}$ long test section, a $6 \mathrm{~m}$ long fetch upwind of the test section, a cross-sectional area of $1 \mathrm{~m} \times 1 \mathrm{~m}$ and operates in suction mode. It has
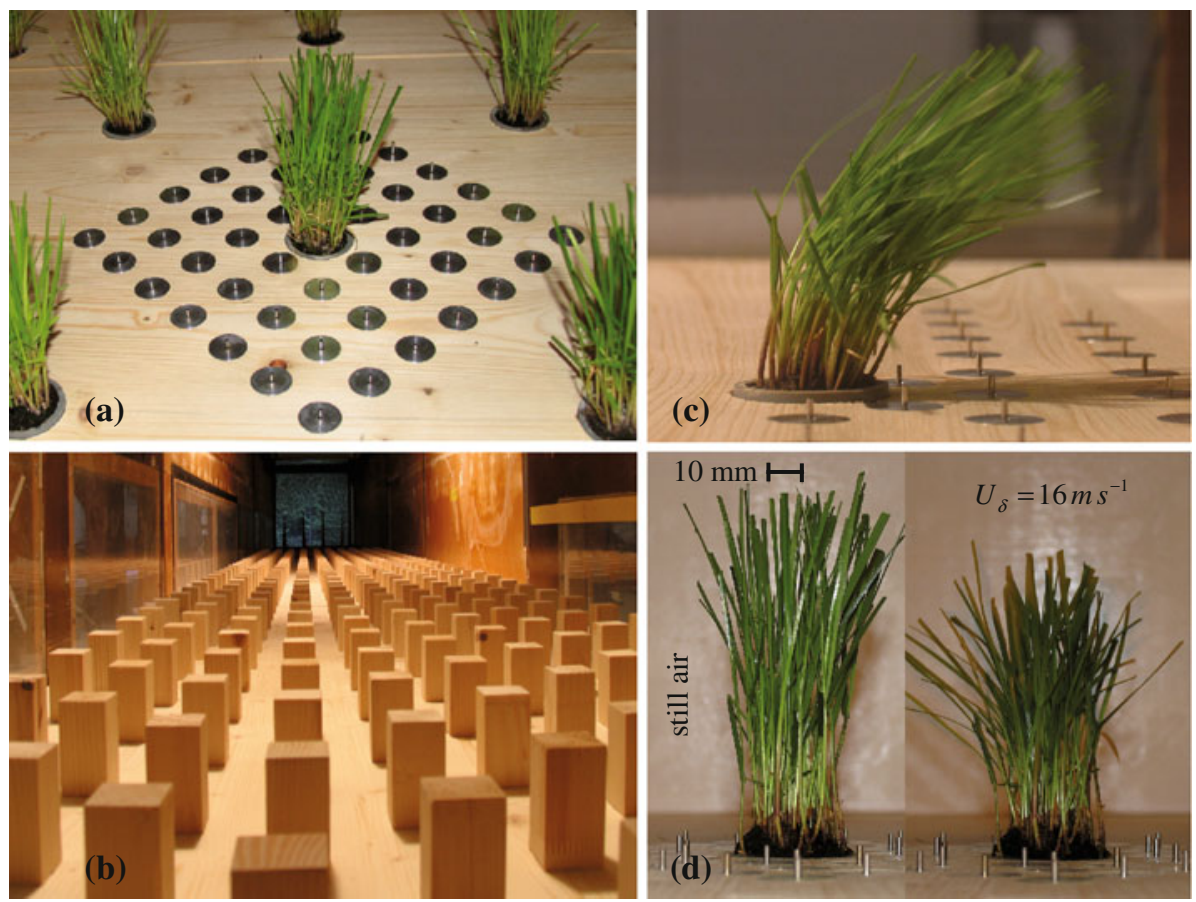

Fig. 1 a Flush mounted Irwin sensors in a live plant canopy (medium density case, $\lambda=0.08$ ), b 8 -m test section covered with cubes (high density case, $\lambda=0.176$ ), $\mathbf{c}$ plant (Lolium perenne) streamlining with the flow (low density case, $\lambda=0.015, U_{\delta}=16 \mathrm{~m} \mathrm{~s}^{-1}$ ), $\mathbf{d}$ front view pictures (streamwise direction) of a plant in still air and for $U_{\delta}=16 \mathrm{~m} \mathrm{~s}^{-1}$ (high density case, $\lambda=0.178$ ) 
Table 1 Experimental summary: (a) number of roughness elements $m^{-2}$, (b) roughness density $\lambda$, (c) freestream velocity $U_{\delta}$ at $z=0.5 \mathrm{~m}$, (d) friction velocity $u_{*}$, (e) average surface shear stress $\tau_{\mathrm{s}}^{\prime}$ on exposed surface area, (f) standard deviation of the spatial variations of $\tau_{\mathrm{s}}(x, y),(\mathrm{g})$ minimum and (h) peak surface shear stress

\begin{tabular}{|c|c|c|c|c|c|c|c|c|}
\hline & \multicolumn{2}{|c|}{$\begin{array}{l}\text { Measurement } \\
\text { set-up }\end{array}$} & \multicolumn{2}{|l|}{ Hotfilm data } & \multicolumn{4}{|c|}{ Irwin sensor data } \\
\hline & & (b) & (c) & (d) & (e) & (f) & (g) & (h) \\
\hline & $\# \mathrm{~m}^{-2}$ & $\lambda$ & $U_{\delta}\left(\mathrm{m} \mathrm{s}^{-1}\right)$ & $u_{*}\left(\mathrm{~m} \mathrm{~s}^{-1}\right)$ & $\tau_{\mathrm{s}}^{\prime}\left(\mathrm{Nm}^{-2}\right)$ & $\sigma\left(\tau_{\mathrm{s}}\right)\left(\mathrm{N} \mathrm{m}^{-2}\right)$ & $\tau_{s, \min }\left(\mathrm{Nm}^{-2}\right)$ & $\tau_{\mathrm{s}}^{\prime \prime}\left(\mathrm{Nm}^{-2}\right)$ \\
\hline \multirow[t]{3}{*}{ Smooth } & 0 & 0 & 8.0 & 0.27 & 0.072 & 0.005 & 0.052 & 0.080 \\
\hline & 0 & 0 & 11.7 & 0.39 & 0.150 & 0.007 & 0.122 & 0.165 \\
\hline & 0 & 0 & 16.3 & 0.57 & 0.252 & 0.010 & 0.215 & 0.277 \\
\hline \multirow[t]{9}{*}{ Plants } & 5.25 & 0.0175 & 8.3 & 0.40 & 0.055 & 0.009 & 0.021 & 0.073 \\
\hline & 5.25 & 0.0166 & 12.1 & 0.56 & 0.128 & 0.018 & 0.065 & 0.158 \\
\hline & 5.25 & 0.0152 & 16.6 & 0.73 & 0.227 & 0.028 & 0.127 & 0.275 \\
\hline & 24.5 & 0.0881 & 7.7 & 0.45 & 0.020 & 0.003 & 0.011 & 0.027 \\
\hline & 24.5 & 0.0879 & 11.4 & 0.64 & 0.047 & 0.006 & 0.028 & 0.062 \\
\hline & 24.5 & 0.0808 & 15.1 & 0.79 & 0.091 & 0.012 & 0.052 & 0.122 \\
\hline & 55 & 0.200 & 8.3 & 0.49 & 0.010 & 0.002 & 0.004 & 0.013 \\
\hline & 55 & 0.189 & 12.6 & 0.70 & 0.020 & 0.002 & 0.013 & 0.024 \\
\hline & 55 & 0.178 & 16.2 & 0.87 & 0.037 & 0.003 & 0.027 & 0.043 \\
\hline \multirow[t]{3}{*}{ Cubes } & 5.25 & 0.0168 & 12.4 & 0.63 & 0.097 & 0.015 & 0.027 & 0.168 \\
\hline & 24.5 & 0.0784 & 12.4 & 0.67 & 0.039 & 0.010 & 0.014 & 0.073 \\
\hline & 55 & 0.176 & 12.3 & 0.65 & 0.026 & 0.005 & 0.012 & 0.037 \\
\hline
\end{tabular}

successfully been used for investigating snow-wind interaction (e.g. Clifton and Lehning 2008 and Gromke et al. 2011) and more recently to investigate soil erosion in live plant canopies (e.g. Burri et al. 2011a,b). An adjustable ceiling allows for the development of a natural boundary layer over various surfaces. The experimental set-up consists of wooden boards in which live plants (Lolium perenne) grown in plastic tubes were arranged in staggered rows (Fig. 1a).

All measurements were performed at the downwind end of the test section. Spires and additional artificial roughness elements were positioned on the fetch upwind of the test section for preconditioning of the boundary-layer flow, as in Burri (2011). Burri (2011) performed a similar study at the SLF wind-tunnel investigating sediment mass flux profiles in live plant canopies of the same densities as investigated in this study. The flow conditioning guarantees the comparability of our shear-stress measurements with results found by Burri et al. (2011b); Burri (2011). With or without flow conditioning on the upwind fetch, the total stress $\tau$ above the medium density canopy case was very similar at the measurement location (Walter et al. 2009).

\subsection{Flow Measurements}

Two-component hot-film measurements using a Dantec (Streamline, sampling frequency $f=20 \mathrm{kHz}$ ) measurement device were carried out to determine basic flow characteristics including vertical profiles of the mean streamwise wind velocity $u$ and the kinematic Reynolds stress $\overline{u^{\prime} w^{\prime}}$. These flow characteristics were used to demonstrate that a well developed natural boundary layer was generated for the different roughness densities and to provide 
a link between the total stress $\tau$ above the roughness elements and the surface shear stress $\tau_{\mathrm{S}}$ measured using Irwin sensors. Following Schlichting (1936), the total shear stress $\tau$ on a rough surface can be split into a component acting on the roughness elements $\tau_{\mathrm{R}}$ and a component acting on the exposed surface area $\tau_{\mathrm{s}}$ so that $\tau=\tau_{\mathrm{s}}+\tau_{\mathrm{R}}$. Vertical hot-film profiles (each with 29 levels) were measured at three different locations for each cube density and for the smooth floor case to obtain one spatially-averaged vertical profile for each roughness density. One profile was measured in the speed-up zone beside, one directly above and one in the wake area downwind of a roughness element. For the plants, eight vertical profiles were measured for the low, seven for the medium, and five for the high density cases at the same locations as mentioned before and at some additional locations. A summary of the hot-film measurement results is given in Table 1 .

\subsection{Surface Shear-Stress Sensors}

Irwin sensors were mounted flush with the surface in an array surrounding a roughness element (Fig. 1a) to determine the spatial and temporal variation of the shear stress $\tau_{\mathrm{s}}(t, x, y)$ (Irwin 1981; Wu and Stathopoulos 1993). The pressure difference $\Delta p$ measured at the Irwin sensor was calibrated against the friction velocity $u_{*}$ in the constant-stress layer measured with the two-component hot-film anemometer. For the smooth wind-tunnel floor, $u_{*}$ is equal to the skin friction velocity $u_{\tau}=\left(\tau_{\mathrm{s}} / \rho\right)^{1 / 2}$; a universal calibration function $u_{\tau}=f(\Delta p)$ was determined for all 32 Irwin sensors used in this study with the calibration showing excellent correlation with Irwin's original calibration (Walter et al. 2011). A custom-made 32-channel pressure scanner (range: $\pm 100 \mathrm{~Pa}$; accuracy: \pm 0.2 Pa typically; sampling rate: $200 \mathrm{~Hz}$ ) was used to measure the pressure difference $\Delta p$. Each Irwin sensor was connected by urethane tubing (length: $0.51 \mathrm{~m}$; inner diameter: $1.65 \mathrm{~mm}$ ) to one transducer of the pressure scanner for the measurements.

To estimate the influence of measurement errors on the surface shear-stress variations, all 32 Irwin sensors with a sensor tube height of $h_{\mathrm{s}}=5 \mathrm{~mm}$ were simultaneously operated at free-stream velocities $U_{\delta}=2-16 \mathrm{~m} \mathrm{~s}^{-1}$ on the smooth wooden wind-tunnel floor without roughness elements (Fig. 2a). The variations averaged $\sigma\left(\tau_{\mathrm{s}}\right) / \tau_{\mathrm{s}}=0.05$ for intermediate to
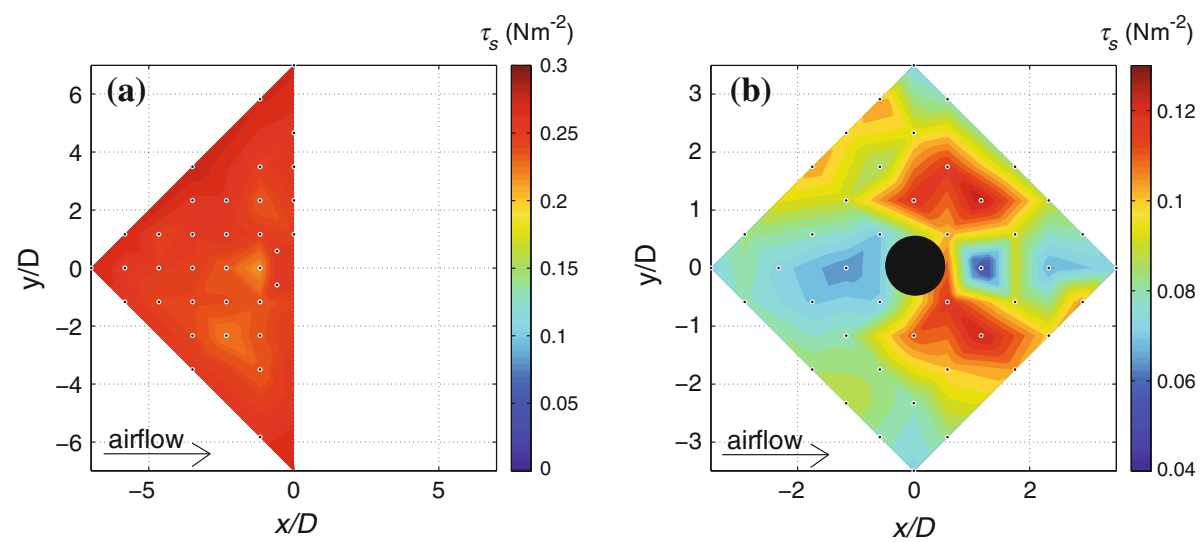

Fig. 2 Temporally-averaged shear-stress distribution $\tau_{\mathrm{s}}(x, y)$ for a the smooth floor case, where slight variations approximate the measurement uncertainty $\left(U_{\delta}=16 \mathrm{~m} \mathrm{~s}^{-1}, \lambda=0, D=40 \mathrm{~mm}\right)$, and $\mathbf{b}$ the medium plant canopy density for a single measured case without averaging for measurement repetitions and for streamwise symmetry $\left(U_{\delta}=16 \mathrm{~m} \mathrm{~s}^{-1}, \lambda=0.088, D=40 \mathrm{~mm}\right)$ 
high surface shear stress $\left(0.017 \mathrm{~N} \mathrm{~m}^{-2}<\tau_{\mathrm{s}}<0.25 \mathrm{Nm}^{-2}\right)$ with a strong increase in variability at very low shear stress $\left(\tau_{\mathrm{s}}<0.017 \mathrm{~N} \mathrm{~m}^{-2}\right)$. Thus, moderate to high free-stream velocities $\left(U_{\delta}=8-16 \mathrm{~m} \mathrm{~s}^{-1}\right)$ were chosen for the experiments to obtain measurable surface shear-stress variations even in the highest roughness density cases. More details about the experiment set-up, the Irwin sensor calibration and the measurement accuracies can be found in Walter et al. (2011).

\subsubsection{Spatial Resolution and Data Processing}

The spatial resolution of the Irwin sensor measurements was $46 \mathrm{~mm} \times 46 \mathrm{~mm}$ for the unplanted and the low density case, $33 \mathrm{~mm} \times 33 \mathrm{~mm}$ for the medium and $25 \mathrm{~mm} \times 25 \mathrm{~mm}$ for the high density case (e.g. Fig. 1a). Due to the limited number of 32 pressure transducers available, it was not possible to measure the surface shear stress at every grid position. Higher measurement densities were chosen close to the roughness elements because the highest spatial variations of the surface shear stress were observed in these regions in preliminary experiments. The positions of Irwin sensor measurements are marked as black or white dots and the wind direction is from the left to the right in all figures. The measured shear-stress data were linearly interpolated onto a regular grid that is assumed to represent the pattern around every plant in the canopy at the measurement section. The location of the roughness element is a singularity with a high spatial variability of the surface shear stress in close proximity to it. A sensitivity study showed no significant difference between the surface shear-stress distributions at the roughness element to surface interface when assuming different conditions at the roughness element location, e.g. $\tau_{\mathrm{s}}=\tau_{\mathrm{s} 0}$ (average $\tau_{\mathrm{s}}$ in the absence of roughness elements) or $\tau_{\mathrm{s}}=0$. Any of these alternatives result in physically questionable representations of $\tau_{\mathrm{s}}$ at that interface when considering the very high spatial variability of the surface shear stress in that area together with our measurement resolution. For simplicity, the shear stress was interpolated across that part of the grid and the values in that location were discarded.

Figure $2 \mathrm{~b}$ shows the raw temporally-averaged (over $30 \mathrm{sec}$ ) shear-stress distribution for a measurement of the medium density plant canopy at $U_{\delta}=16 \mathrm{~m} \mathrm{~s}^{-1}$. Variations in the experiment set-up and the measurement technique result in a distribution that shows slight deviations from streamwise symmetry for the left $(y / D>0)$ and the right $(y / D<0)$ halves of the measurement grid. Here, $D$ is the average plant or cube diameter. In all other presentations of these data, the left and the right half (streamwise sides) were averaged to obtain symmetry and thus a clearer picture of the systematic patterns. Additionally, all figures (except those in Fig. 2) are an average of three measurement repetitions, each with different plants in the positions around the Irwin sensors to account for slight variations in plant shape that might affect the shear-stress distribution.

\subsubsection{Temporally-Resolved Surface Shear-Stress Data}

Measuring the surface shear stress at a frequency of $200 \mathrm{~Hz}$ allows for statistical analysis of temporal surface shear-stress variations. Irwin (1981) found a good correlation between velocity time series simultaneously measured with an Irwin sensor and a hot-wire anemometer at the same location (at the Irwin sensor tube height $h_{\mathrm{s}}$ ). The tube connecting their Irwin sensor to a pressure transducer was $0.61 \mathrm{~m}$ long (similar to our set-up). In the spectral domain, the magnitudes of the Irwin sensor velocities agreed within $\pm 10 \%$ with the hot-wire measurements in a range from $f=0$ to $80 \mathrm{~Hz}$. The combination of the original Irwin calibration 
function for wind velocities at the sensor tube height $U_{s}=f(\Delta p)$ with that for the skin friction velocity $u_{\tau}=f(\Delta p)$ (Irwin 1981) involves a linear relation $u_{\tau}=f\left(U_{s}\right) \approx c_{1} U_{s}+c_{2}$ where $c_{1}$ and $c_{2}$ are constants. The magnitudes of our skin friction velocity time series $u_{\tau}(t)$ are thus assumed to be correct within the same range of accuracy of $\pm 10 \%$. The damping of the signal induced by the tubing resulted in a steadily increasing phase shift of the signals relative to the hot-wire measurements with increasing frequency (Irwin 1981). Our pressure time series $\Delta p(t)$ measured at the Irwin sensors, each $\Delta T=30 \mathrm{~s}$ long, were digitally lowpass filtered with a cut-off frequency of $f_{\mathrm{c}}=80 \mathrm{~Hz}$.

Irwin (1981) defined the standard deviation of the wind speed $u(t)$ at the height of the Irwin sensor tube as

$$
\sigma_{u}=\sqrt{\overline{u^{\prime 2}}}=\frac{\beta_{\mathrm{c}}}{2} \frac{\sqrt{\overline{\Delta p^{\prime 2}}}}{\sqrt{\overline{\Delta p}}},
$$

where $\beta_{\mathrm{c}}$ is a calibration constant arising from the calibration of the Irwin sensors against wind speed and $\Delta p^{\prime}$ is the pressure fluctuation around the mean value. Note that Irwin sensor measurements contain no information on the direction of the airflow, so the wind speed $u(t)$ is not the streamwise velocity component in the case of Irwin sensor measurements. Sutton and McKenna-Neumann (2008) defined a measure $\xi$, which they referred to as a normalized turbulence intensity, and which gives the relative increase or decrease in the standard deviation $\sigma_{\mathrm{u}}$ introduced by the roughness elements relative to the smooth floor case:

$$
\xi=\frac{\sigma_{\mathrm{u}, \mathrm{R}}}{\sigma_{\mathrm{u}, \mathrm{s}}} .
$$

The local skin friction velocity $u_{\tau}(t, x, y)$ on the ground beneath the non-erodible roughness elements is not constant with time and may (or may not) exceed a fluid threshold skin friction velocity $u_{\tau t}$, above which particle erosion begins for some fraction of time even if the average surface shear-stress velocity $u_{\tau}$ is smaller than $u_{\tau t}$. The percentage of time $\Delta t$ while the surface shear-stress velocity $u_{\tau}>u_{\tau t}$ can be used to assess the local predominance of erosion and deposition mechanisms:

$$
\psi(x, y)=\frac{\Delta t\left(u_{\tau}(t, x, y)>u_{\tau t}\right)}{\Delta T} .
$$

Note that the fluid threshold $u_{\tau t}$ defined here is greater than the average fluid threshold friction velocity $u_{*_{t}}$ measured in the constant-stress layer at the commencement of particle erosion. The reason for this is that the peak values of $u_{\tau}(t, x, y)$, not the spatiotemporal average, are responsible for the onset of particle erosion. The procedure to estimate $u_{\tau t}$ is described in Sect. 3.4.2.

\section{Results and Discussion}

\subsection{Flow Characteristics}

Figure 3 shows the vertical profiles of the normalized mean streamwise wind velocity $u$ and the normalized kinematic Reynolds stress $\overline{u^{\prime} w^{\prime}}$ for the different roughness configurations at the reference free-stream velocity $U_{\delta}=12 \mathrm{~m} \mathrm{~s}^{-1}$. Each of the vertical profiles represents a spatial average of at least three and up to eight two-component hot-film profiles measured at different locations above the roughness elements. 

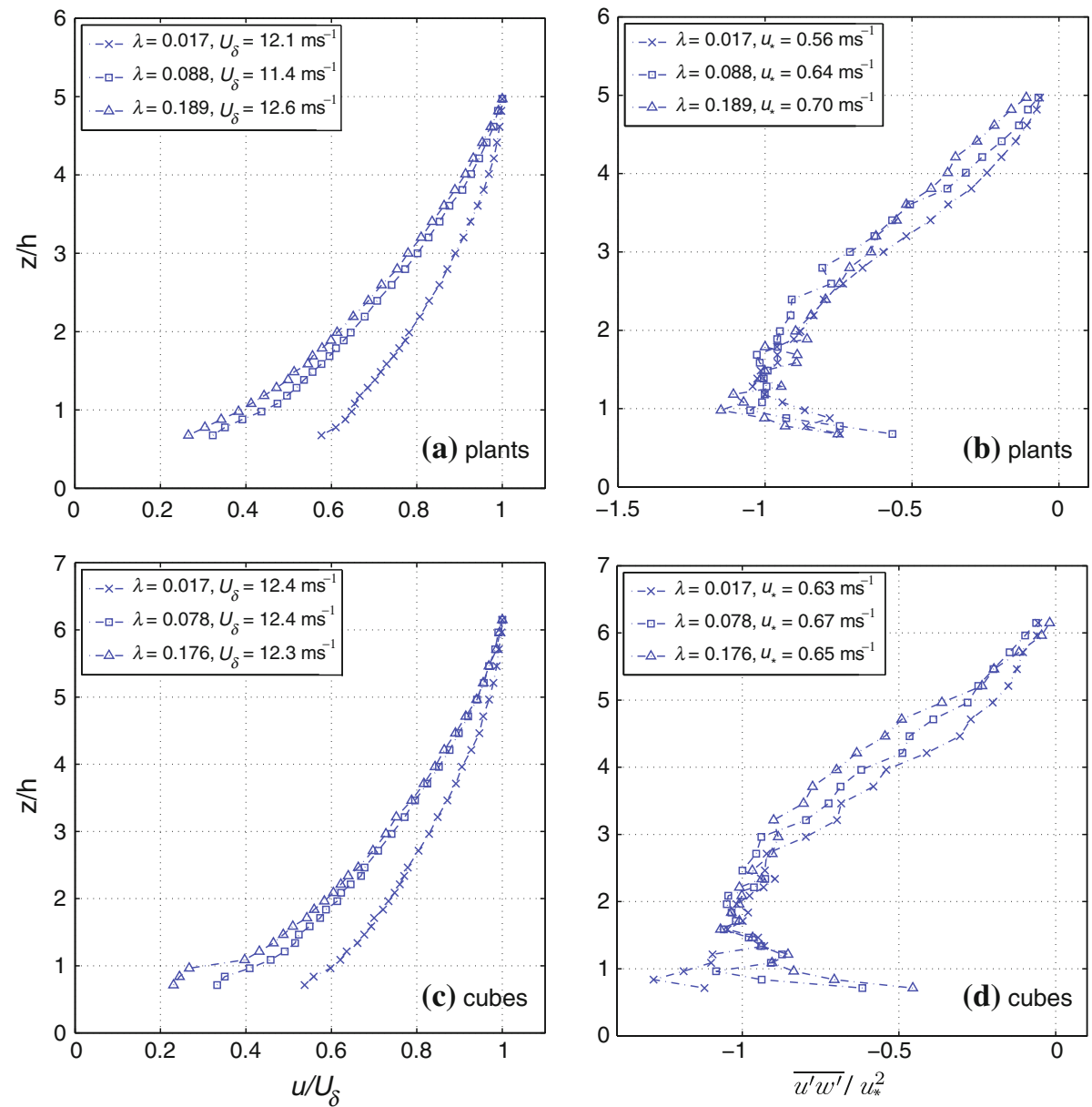

Fig. 3 Normalized mean wind velocity $u$ and kinematic Reynolds stress $\overline{u^{\prime} w^{\prime}}$ profiles: a, $\mathbf{b}$ for the plant experiments, $\mathbf{c}, \mathbf{d}$ for the cube experiments. $U_{\delta}=12 \mathrm{~m} \mathrm{~s}^{-1} ; h=0.1 \mathrm{~m}$ for the plant and $h=0.08 \mathrm{~m}$ for the cube cases

The mean wind velocities directly above the roughness elements $u(z / h=1)$ were similar for the plant and the cube experiments at each roughness density $\lambda$ (Fig. 3a, c). This demonstrates that flow conditions were comparable in the two cases, allowing comparison of the surface shear-stress distributions on the ground beneath the plants and the cubes. Plants streamline with the flow at higher wind velocities, resulting in aerodynamical shapes, reduced flow resistances of the plants, and thus in slightly higher wind velocities $u(z / h=1)$ compared to the cubes as shown in Fig $3 \mathrm{a}$ and $\mathrm{c}$. The mean velocity profile $u(z)$ for the high density cube case shows a strong inflection near the top of the cubes consistent with a skimming flow regime where the flow above the roughness elements is weakly coupled with the flow among the roughness elements (Morris 1955). In the high density plant case the inflection is not clearly visible. This may be due to the fluctuating motions of the plants and their porous structure that may cause a weakening or even a suppression of flow separation around the plants. The low and the medium roughness densities are considered to be cases of isolated roughness and wake interference flow, and the associated shear-stress distributions will be discussed in the following section. 
While keeping the free-stream velocity constant, the friction velocity in the constant-stress layer $u_{*}$ increases steadily from $u_{*}=0.56 \mathrm{~m} \mathrm{~s}^{-1}$ in the low to $u_{*}=0.70 \mathrm{~m} \mathrm{~s}^{-1}$ in the high density plant case (Table 1). For the cubes, $u_{*}=0.67 \mathrm{~m} \mathrm{~s}^{-1}$ for the medium density, but decreases to $u_{*}=0.65 \mathrm{~m} \mathrm{~s}^{-1}$ for the high density case suggesting a slight decrease in flow resistance induced by the high cube density due to the skimming flow regime. However, $u_{*}$ is larger for the plants than for the cubes in the high density case suggesting an increase in flow resistance induced by the plants fluctuating motion. For both the plant and the cube cases, a maximum in the absolute values of the kinematic Reynolds-stress profiles was found directly above the roughness elements at $z / h \approx 1$ and remained nearly constant up to $z / h \approx 2$ in the plant and $z / h \approx 2.5$ in the cube case, similar to constant-stress layers found in the neutral boundary layer.

\subsection{Shear-Stress Partitioning}

A common way to quantify the sheltering effect of non-erodible roughness elements is to determine the stress partition $\left(\tau_{\mathrm{s}} / \tau\right)^{1 / 2}$, i.e. the surface shear-stress divided by the total stress above the canopy (Eq. 1). Numerous wind-tunnel and field investigations have provided data on shear-stress partitioning by various types of roughness elements, roughness densities and roughness configurations. Figure 4 shows a summary of some of these results along with our new measurements. These include the shear-stress partition for the average surface shear-stress on the exposed surface area $\left(\tau_{\mathrm{s}}^{\prime} / \tau\right)^{1 / 2}$ (Fig. 4a) and the peak surface shear-stress $\left(\tau_{\mathrm{S}}^{\prime \prime} / \tau\right)^{1 / 2}$ (Fig. 4b). Good agreement was found between our data and these earlier studies, demonstrating that the Irwin sensors produce reliable surface shear-stress data. Both the average and the peak shear-stress on the surface decrease with increasing roughness densities $\lambda$ as expected, with notable differences between the plant and cube experiments.

Because plants streamline with the flow, their frontal area $A_{\mathrm{f}}$ and thus the roughness density $\lambda$ decrease with increasing wind speeds and become a function of the Reynolds number as demonstrated by Gillies et al. (2002) (Fig 1c, d). The frontal area $A_{\mathrm{f}}$ first increases slightly by about $3 \%$ relative to $A_{\mathrm{f}} \approx 0.0033 \mathrm{~m}^{2}$ in still air between $U_{\delta}=4-8 \mathrm{~m} \mathrm{~s}^{-1}$ because the plants flutter and expand in low to moderate winds. At higher wind velocities $A_{\mathrm{f}}$ decreases because the plants streamline with the flow, reaching about $90 \%$ of their still-air frontal area at $U_{\delta}=16 \mathrm{~m} \mathrm{~s}^{-1}$. The percentage increase and decrease of $A_{\mathrm{f}}$ are average values from
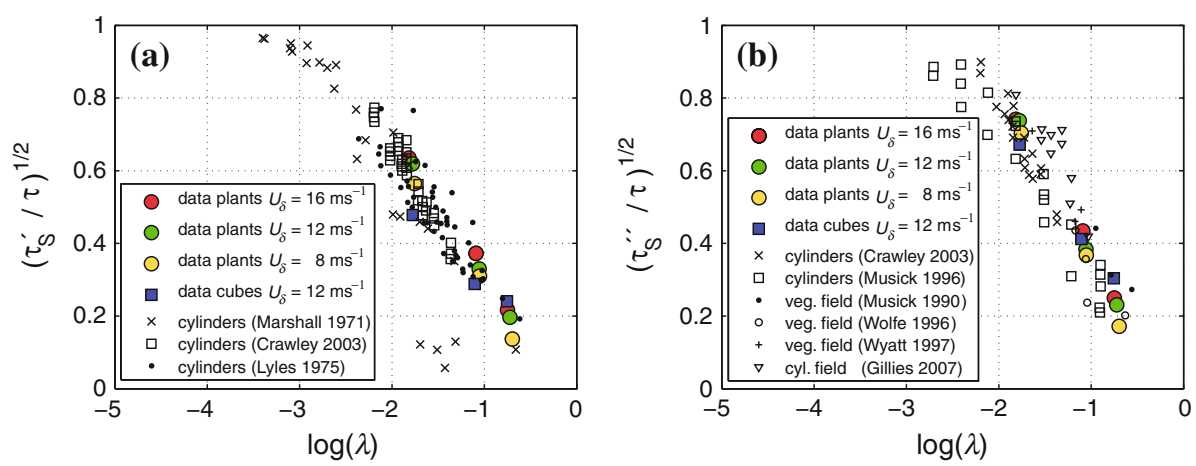

Fig. 4 Comparison of measured surface shear-stress ratios with literature data: a average surface shear-stress on exposed surface area $\tau_{\mathrm{s}}^{\prime}$ and $\mathbf{b}$ peak surface shear stress $\tau_{\mathrm{s}}^{\prime \prime}$. Literature values measured in field experiments are indicated with "field" whereas the remaining data points were obtained by wind-tunnel measurements 
multiple plants and several plant photographs, since the plant waving motion results in rapidly changing frontal areas. No significant differences in the change of $A_{\mathrm{f}}$ with wind speed were found for the three different roughness densities. This streamlining of the roughness elements results in higher wind speeds close to the ground and thus in increased average $\left(\tau_{\mathrm{s}}^{\prime}\right)$ and peak $\left(\tau_{\mathrm{s}}^{\prime \prime}\right)$ surface shear stress at the ground. The three data points for each plant canopy density $\lambda$ in Fig. 4 correspond to three different free-stream velocities $\left(U_{\delta}=8,12\right.$ and $16 \mathrm{~m} \mathrm{~s}^{-1}$ ) and thus to three different Reynolds numbers defined as $R e_{\mathrm{h}}=U_{\delta} h / v$ where $v=1.5 \times 10^{-5} \mathrm{~m}^{2} \mathrm{~s}^{-1}$ is the kinematic viscosity of air and $h=103,99$ and $94 \mathrm{~mm}$ the average height of the plants at $U_{\delta}=8,12$ and $16 \mathrm{~m} \mathrm{~s}^{-1}$, respectively. Figure 4 shows that with increasing $R e_{\mathrm{h}},\left(\tau_{\mathrm{s}}^{\prime} / \tau\right)^{1 / 2}$ and $\left(\tau_{\mathrm{s}}^{\prime \prime} / \tau\right)^{1 / 2}$ also increase.

Both the average and peak shear-stress partitions are larger for the plants than the cubes at the low roughness density $(\log (\lambda) \approx-1.7)$. The opposite is true at the high roughness density $(\log (\lambda) \approx-0.7)$, where the cubes result in larger shear-stress partitions. We hypothesize that in the high density plant canopy the flexible plants bend to cover a larger fraction of the surface, resulting in very low surface shear stress. In the high density cube case, the flow penetrates slightly deeper into the canopy resulting in higher surface shear stresses than in the plant case. Additionally, the total shear stress $\tau$ is lower for the high density cube case than for the high density plant case as discussed before in Sect. 3.1. This demonstrates that the shear-stress partition depends strongly on the type of roughness element used and that results based on rigid and non-porous roughness elements may not be adequate for the evaluation of erosion and sediment transport in real plant canopies.

\subsection{Surface Shear-Stress Spatial Variability}

The shear-stress partitions from the previous section are either spatial averages $\left(\tau_{\mathrm{s}}^{\prime} / \tau\right)^{1 / 2}$ or point data $\left(\tau_{\mathrm{s}}^{\prime \prime} / \tau\right)^{1 / 2}$ that might be used to estimate the integral total mass flux of sediment transport above a vegetation canopy or to determine the onset of particle erosion. Estimation of these quantities is a clear practical application of this study. However, for more precise modelling of sediment mass fluxes or the patterns of erosion and deposition expected on a partially-sheltered surface, the spatial and temporal variability of the surface shear stress $\tau_{\mathrm{s}}(t, x, y)$ must also be known. Figure 5 shows the spatial patterns of the surface shear-stress partition $\left(\tau_{\mathrm{s}}(x, y) / \tau\right)^{1 / 2}$ for the plants (left column) and cubes (right column). The shearstress scaling at each canopy density is the same for the plant and cube cases and the lower limit is the same for all plots. The $x$ - and $y$-axes were normalized by the cube side length and the average plant diameter, which are both $D=40 \mathrm{~mm}$.

Several features of the mean flow around the roughness elements can be inferred from the stress distributions shown in Fig. 5. The low shear stress measured directly upwind of the roughness elements indicates the presence of a flow stagnation zone with reduced wind speeds. Within the wake region downwind of the roughness element, flow separation and reattachment result in a sheltered area with the lowest surface shear stress. The flow convergence beside the roughness elements results in speed-up zones characterized by the largest surface shear stress. Sutton and McKenna-Neumann (2008) reported that rigid and non-porous roughness elements with well-defined flow separation at the edges result in the development of flow structures similar to horseshoe vortices wrapping around and paired counter-rotating eddies with a vertical axis immediately in the lee of the roughness element. Our plants consist of various small stems and blades shedding much smaller eddies than the cubes in diverse directions, suggesting that flow structures such as horseshoe vortices and counter-rotating eddies may be less organized and coherent for vegetation than for the rigid cylinders used in their 

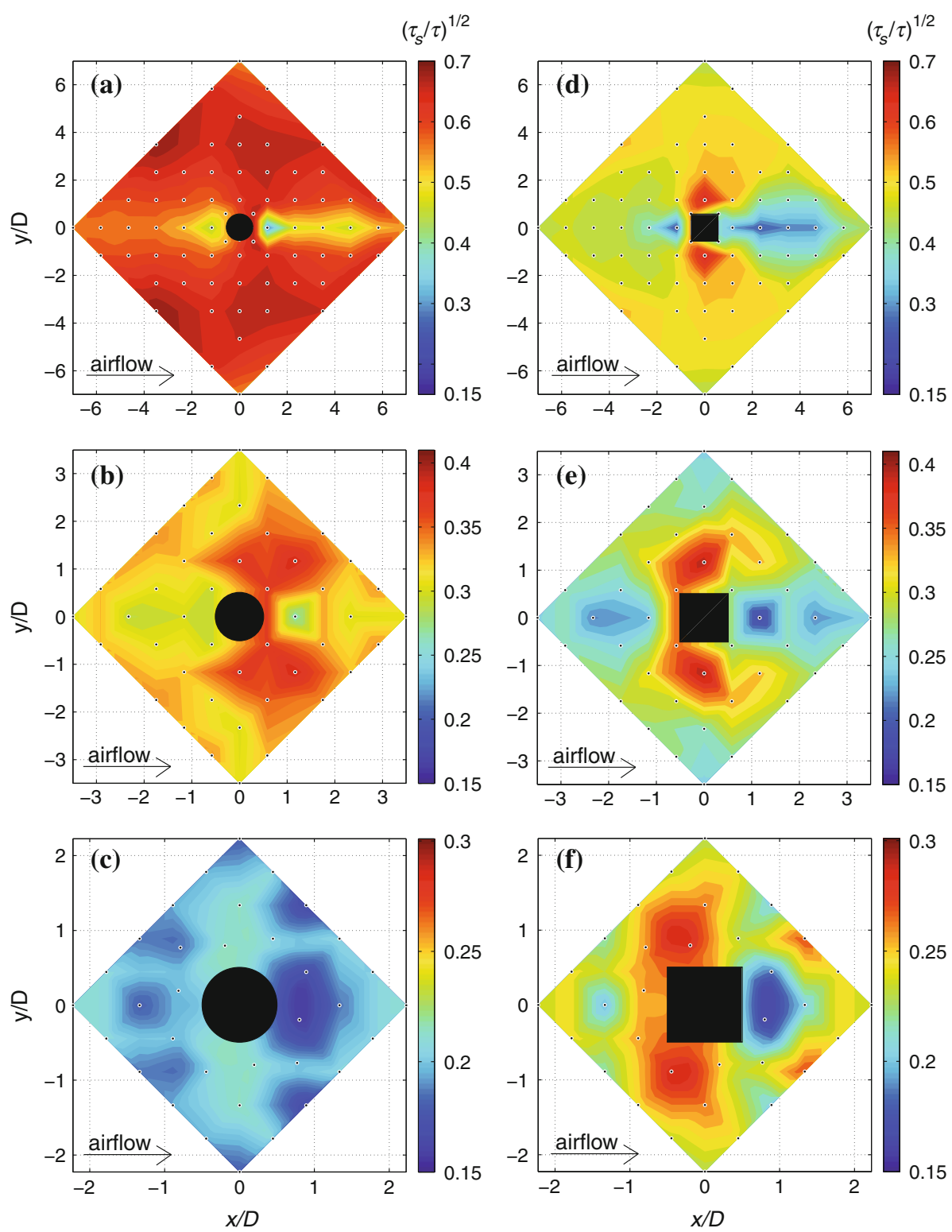

Fig. 5 Spatial distributions of the shear-stress partition $\left(\tau_{\mathrm{S}}(x, y) / \tau\right)^{1 / 2}$ for the three different roughness densities: $\mathbf{a}-\mathbf{c}$ plants and $\mathbf{b}-\mathbf{f}$ cubes for the low/medium/high roughness density cases at $U_{\delta}=12 \mathrm{~m} \mathrm{~s}^{-1}(D=40 \mathrm{~mm})$

study. Increased shear stress directly in front and behind the roughness elements (strongest in Fig. 5b, e) are interpolation artefacts that can be explained by the measurement resolution.

As mentioned in the previous section, results from different roughness densities represent different flow regimes. The sheltered areas in both low roughness density cases do not reach the next roughness element downstream, so the low-density configuration in both 
cases (Fig. 5a, d) represents isolated roughness flow as defined by Morris (1955). In the medium density cases, the sheltered area reaches the next roughness element downstream while a significant fraction of the surface remains unsheltered, so these cases (Fig. 5b, e) are good examples of wake interference flow. For the high density plant case, the surface is almost completely sheltered suggesting a skimming flow regime (Fig. 5c). For the high density cube case, a maximum shear-stress partition of $\left(\tau_{\mathrm{s}}(x, y) / \tau\right)^{1 / 2} \approx 0.3$ was found in the speed-up zones, suggesting that the surface was not completely sheltered (Fig. 5f). However, the strong inflection found for the $u(z)$ profiles in Sect. 3.1 indicates that this case was also a skimming flow regime. Comparing the surface shear-stress distributions for the plants with those for the cubes shows strong differences between the two, suggesting that a different sheltering effect can be expected from plants than from rigid roughness elements. This suggests that different local as well as total erosion and deposition rates can be expected, resulting in different erosion patterns and total mass fluxes.

Solid and inflexible roughness elements such as cubes generate a stronger lateral deflection of the airflow than do plants, resulting in higher flow speeds along their sides. This is substantiated by the fact that higher peak shear-stress values $\tau_{\mathrm{s}}^{\prime \prime}$ were found for the cubes than for the plants at all roughness densities (Table 1). Since the peak shear stresses are responsible for the initiation of soil erosion, our results suggests that plants provide a better sheltering effect than cubes or other more natural rigid roughness elements such as stones. However, the fact that the total stresses $\tau$ in the cube experiments are also larger than for the plants results in similar peak shear-stress partition values for the low and medium roughness densities (Figs. 4b, 5a,b,d,e). This is why the peak shear-stress partitions $\left(\tau_{\mathrm{s}}^{\prime \prime} / \tau\right)^{1 / 2}$ for the plants, cubes and other roughness elements from the literature are all within the same range (Fig. 4b). The stronger flow deflections around rigid obstacles result in higher peak surface shear stresses as well as higher total shear stress above the roughness elements due to increased flow resistance imposed by rigid rather than porous and flexible roughness elements.

The sheltered areas in the lee of the plants are significantly narrower with higher shearstress ratios $\left(\tau_{\mathrm{s}} / \tau\right)^{1 / 2}$ than the cubes. This is most evident in Fig. 5a, d. The streamlining of the plants results in increasingly narrow sheltered areas at higher wind velocities. This can be shown by comparing the surface shear stress in the planted cases with the surface shear stress in the absence of any roughness elements $\tau_{\mathrm{s} 0}$ as $\left(\tau_{\mathrm{s}}(x, y)-\tau_{\mathrm{s} 0}\right) / \tau_{\mathrm{s} 0}$ at different free-stream velocities $U_{\delta}$ (not shown here). For the cubes, however, these $\left(\tau_{\mathrm{s}}(x, y)-\tau_{\mathrm{s} 0}\right) / \tau_{\mathrm{s} 0}$ plots are identical at different $U_{\delta}$, suggesting a Reynolds-independent flow. The Reynolds number $R e_{\mathrm{h}}$ for the cube cases varies between 42,000 and 85,000 with a consistent cube height $h=80 \mathrm{~mm}$. In this range, the drag coefficient of cylinders or cubes are Reynoldsnumber independent (Schlichting 1936) confirming our finding that the sheltering effect of the cubes is not dependent on the speed of the flow.

Solid circular cylinders may have provided a better representation of real plants, since their bulk shape is more similar to that of a plant than the cube shape. However, preliminary experiments comparing the surface shear stress on the ground beneath a single live plant and a single circular cylinder of similar size show differences comparable to those found between the plant canopies and the cube arrays, e.g. larger shelter areas and higher peak stress values $\tau_{\mathrm{s}}^{\prime \prime}$ for the cylinder. Furthermore, the cylinder was found to result in even higher $\tau_{\mathrm{s}}^{\prime \prime}$ values than the cubes. This can be explained by the flow separation at the vertical windward edges of a cube that results in higher turbulence generated and less speed-up of the flow than around a cylinder with a more streamlined shape. This finding suggests that in some cases, cubes may actually be better representations of live plants than cylinders. 


\subsection{Time Series Statistics}

\subsubsection{Turbulence Intensities}

The normalized turbulence intensity distributions $\xi(x, y)$ (Eq. 3) for the low roughness density cases shown in Fig. 6 display the increase or decrease in $\sigma_{\mathrm{u}}$ caused by the roughness elements relative to the smooth floor case. Higher normalized turbulence intensities $\xi$ were found at all roughness densities in experiments with cubes than those with plants. The peak values of the normalized turbulence intensity $\xi$ for the plants were found in the wake areas and are consistent from the low to the high density case with an increase in $\sigma_{\mathrm{u}}$ of about $40 \%(\xi=1.4)$ relative to the smooth floor case. In contrast, the peak values for $\xi$ in the cube cases $(\xi=2.2$ for the low, $\xi=1.7$ for the medium and $\xi=1.5$ for the high density) are mainly found upwind and at the sides of the cubes, a region that matches the horseshoe vortices observed by Sutton and McKennaNeumann (2008) (Fig. 6b). This suggests that the high turbulence intensities $\xi$ in the cube cases may result from horseshoe vortices. Furthermore, the low values of $\xi$ in the plant cases supports the hypothesis that horseshoe vortices are suppressed as suggested in Sect. 3.3.

Plants appear to induce lower turbulence levels close to the ground than cubes due to the streamlining behaviour of the plants resulting in more favourable aerodynamical shapes. The lower near-ground turbulence intensities also support our finding that plants provide a better sheltering effect than rigid roughness elements. It is remarkable that even for the low near-ground mean wind velocities in the high density plant and cube case, $\sigma_{\mathrm{u}, \mathrm{R}}$ is everywhere still larger than $\sigma_{\mathrm{u}, \mathrm{s}}$, e.g. $\xi>1$ (not shown here). These results agree with those of Sutton and McKenna-Neumann (2008). Values of $\xi$ slightly lower than one are found directly upwind of the plants in the low density case. Despite the increased turbulence intensities found in the presence of roughness elements, the horizontal velocity variations $u^{\prime}$ in the sheltered area downwind of the roughness elements seem to be poorly correlated with the vertical velocity fluctuations $w^{\prime}$. This is due to a reduced downward momentum flux and thus reduced surface shear stress found in the wake area of the roughness elements (see Figs. 5, 6).
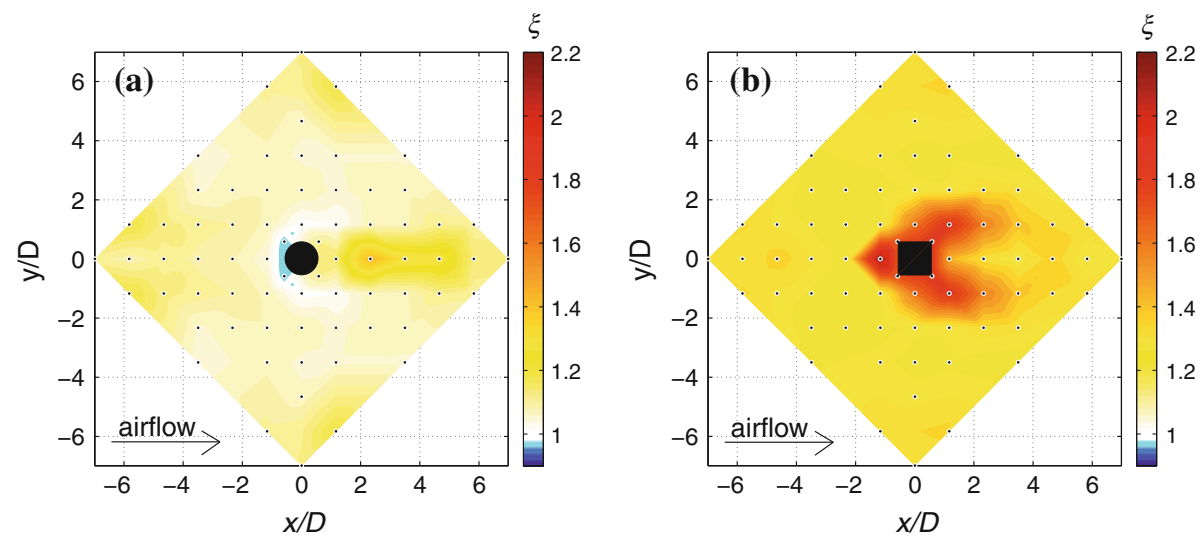

Fig. 6 Normalized turbulence intensity distributions $\xi(x, y)$ showing the increase in $\sigma_{\mathrm{u}}$ close to the ground relative to the smooth floor case: $\mathbf{a}$ plants and $\mathbf{b}$ cubes (low roughness density, $U_{\delta}=16 \mathrm{~m} \mathrm{~s}^{-1}, D=40 \mathrm{~mm}$ ) 


\subsubsection{Threshold Friction Velocity}

The spatial patterns of the percentage of time $\psi(x, y)$ (Eq. 4) that a fluid threshold skin friction velocity $u_{\tau t}=0.35 \mathrm{~m} \mathrm{~s}^{-1}$ was exceeded during our experiments show interesting similarities to patterns of erosion and deposition found in an earlier study at the SLF wind tunnel (Burri et al. 2011b; Burri 2011). Burri et al. (2011b) investigated the vertical profiles of drifting sediment mass flux in live plant canopies of various densities. The sediment used in that study was a commercial quartz sand with grain diameters between $d=0.4-0.8 \mathrm{~mm}$. Those authors found a fluid threshold friction velocity of $u_{*_{t}}=0.3 \mathrm{~m} \mathrm{~s}^{-1}$ (at $U_{\delta}=8 \mathrm{~m} \mathrm{~s}^{-1}$ ) at the onset of erosion for an unplanted sand surface. We calculate their fluid threshold skin friction velocity (see Sect. 2.2.2) as $u_{\tau t}=u_{*_{t}}+2 \sigma\left(u_{\tau}\right)$, where the standard deviation $\sigma\left(u_{\tau}\right)=0.025 \mathrm{~m} \mathrm{~s}^{-1}$ at $U_{\delta}=8 \mathrm{~m} \mathrm{~s}^{-1}$ was determined from the temporal skin friction velocity variations measured with Irwin sensors on the smooth wooden floor without roughness elements for the present study. By adding $2 \sigma\left(u_{\tau}\right)$ we assume that $5 \%$ of the skin friction velocities that are higher than $u_{*_{t}}+2 \sigma\left(u_{\tau}\right)$ are responsible for the initiation of particle erosion on the unsheltered sand surface.

Figure $7 \mathrm{a}-\mathrm{d}$ shows $\psi(x, y)$ for the low and the medium density plant and cube case at $U_{\delta}=16 \mathrm{~m} \mathrm{~s}^{-1}$. For $\psi=1$, the threshold $u_{\tau t}$ at the surface is exceeded at all times, indicating a region of erosion. For $\psi=0$, the sediment transport threshold was never exceeded, so deposition of incoming particles should always be possible. However, for $0<\psi<1$, erosion and deposition are both possible, and the magnitude of local net erosion or deposition depends on the value of $\psi$. For $\psi=0.5$, the local temporally-averaged skin friction velocity $u_{\tau}$ approximately equals the threshold value $u_{\tau t}=0.35 \mathrm{~m} \mathrm{~s}^{-1}$. Local net deposition might thus be predicted for $\psi<0.5$ and local net erosion for $\psi>0.5$. The parameter $\psi$ was chosen to illustrate local erosion and deposition patterns rather than $u_{\tau}$ because the upper and lower limits for erosion and deposition are clearly defined as $\psi=1$ and $\psi=0$, and because $\psi$ is directly related to the number of erosive events that occur when larger eddies strike the surface.

Figure $7 \mathrm{e}, \mathrm{f}$ are photographs taken with a vertical perspective at the measurement section that show the erosion and deposition patterns of sand for the low and medium plant density cases with illumination from the side (Burri et al. 2011b). Prior to these photographs, a freestream velocity of $U_{\delta}=16 \mathrm{~m} \mathrm{~s}^{-1}$ persisted for approximately $200 \mathrm{sec}$ while drifting sand was present. The erosion and deposition patterns are qualitatively in excellent agreement with the spatial patterns of $\psi$ found in our plant experiments (Fig. 7c, d). The deposited (or undisturbed) sand upwind and in the lee of the plants in the low density plant case (Fig. 7e) corresponds with the areas where $\psi<0.75$ in Fig. 7c. This shows that the suppression of erosion and particle deposition are possible even if the skin friction velocity exceeds the threshold value $u_{\tau t}$ during $75 \%$ of the time. This is likely due to sand particles striking the plant, losing some of their momentum, and being deposited in the wake behind the plant. In the medium density plant experiment (Fig. 7d, f), strong net erosion occurred outside the sheltered area even at low $\psi(0.25<\psi<0.5)$. We hypothesize that this is due to a reduction in threshold skin friction velocity $u_{\tau t}$ after sediment transport has been initiated, as saltating particles can dislodge stationary particles on impact (Bagnold 1943). Therefore, for the case of sand transport in plant canopies discussed here, when $\psi>0.75$ erosion dominates, and for $\psi<0.25$ deposition dominates. In the range $0.25<\psi<0.75$, either may be true. For the high roughness density cases, $\psi<0.05$ was found everywhere in both the plant and the cube cases and the surface was entirely sheltered. This result is supported by the negligibly small total sediment mass flux found for the high density case by Burri et al. (2011b). 

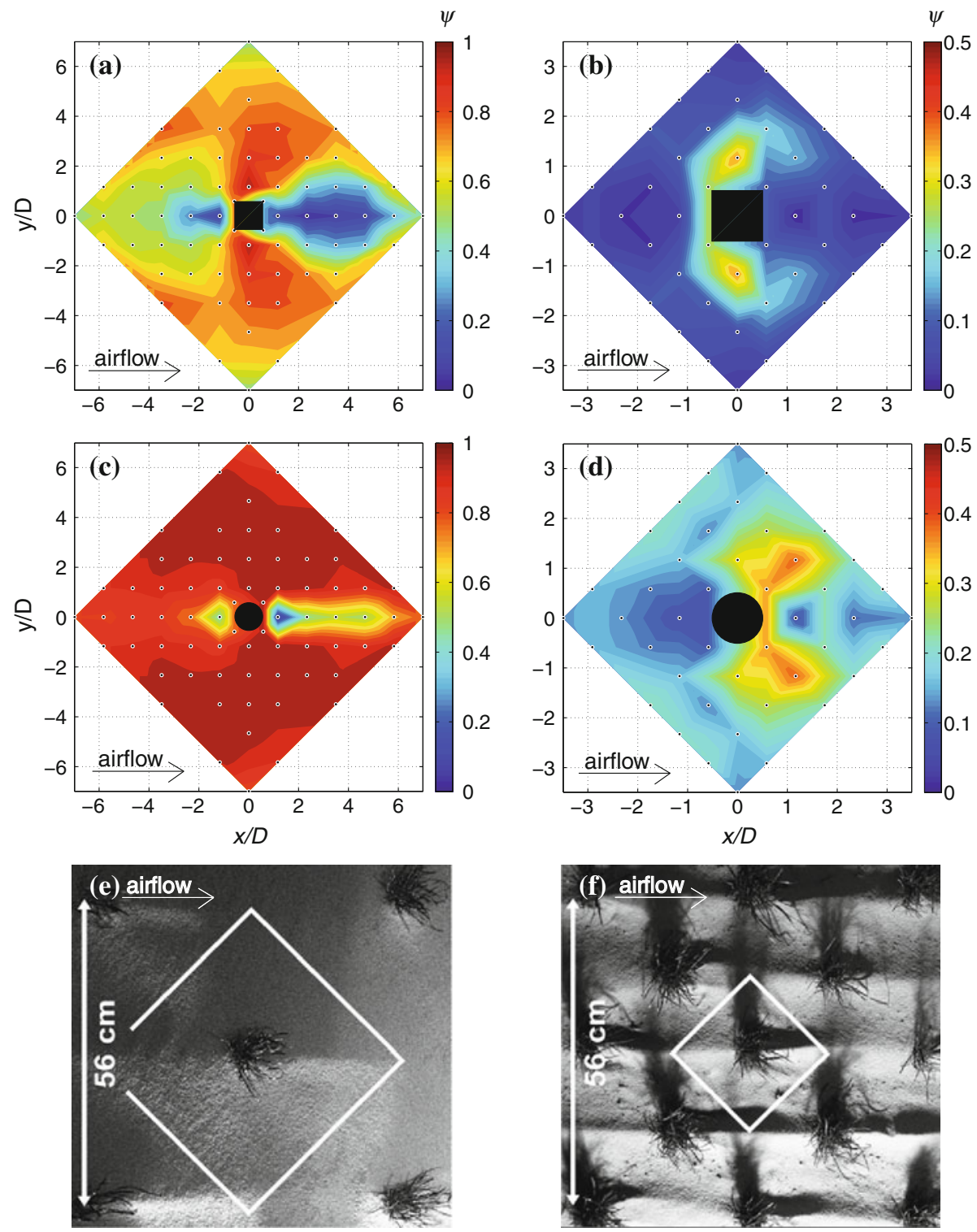

Fig. 7 Percentage of time $\psi$ that a fluid threshold skin friction velocity $u_{\tau t}=0.35 \mathrm{~m} \mathrm{~s}^{-1}$ was exceeded for the low and the medium cube $(\mathbf{a}, \mathbf{b})$ and plant $(\mathbf{c}, \mathbf{d})$ density cases $\left(U_{\delta}=16 \mathrm{~m} \mathrm{~s}^{-1}, D=40 \mathrm{~mm}\right)$. e, f are vertical photographs of plants and corresponding erosion patterns illuminated from the side for the low and medium roughness density of sand erosion experiments in live plant canopies $\left(U_{\delta}=16 \mathrm{~ms}^{-1}\right.$ ) (from Burri et al. 2011a,b)

The strong differences in the spatial patterns of $\psi$ in the cube and the plant cases (Fig. $7 \mathrm{a}-\mathrm{d})$ show that very different erosion patterns and perhaps differences in the total sediment mass flux would be expected in sediment transport experiments using cubes. This supports our assertion that investigations using rigid roughness elements may not provide appropriate data from which to draw conclusions regarding natural plant canopies. 
It should be pointed out that a comparison between sand erosion patterns and surface shear-stress measurements in the absence of drifting sand may not be strictly appropriate. Drifting sand interacts with the boundary layer and causes deceleration of the airflow within the saltation layer (Bagnold 1943). The drifting sand cases may thus have had slightly lower skin friction velocities than those measured with the Irwin sensors in the absence of drifting sand. Also, the topography of the surface changes when erosion and deposition take place, resulting in changes to the surface shear-stress distribution and the local wind field. The good correlation between the patterns of $\psi$ and sediment erosion suggests that, while these limitations might mean that the absolute values of $\psi$ in the two cases were not identical, their spatial distributions were very close.

The above findings suggest that $\psi$ could be a useful parameter for determining local erosion and deposition rates. Furthermore, our results demonstrate the importance of knowing the spatial and temporal variability of the shear stress on the ground, rendering this method somewhat impractical for field studies. Experiments performed by Valyrakis et al. (2010) in a flume with a single coarse sediment grain (diameter: $12.7 \mathrm{~mm}$ ) showed that not only the magnitude but also the duration of high surface shear events defines the onset of particle entrainment. We thus anticipate future work on this issue involving a similar study for smaller sand grains $(0.4-0.8 \mathrm{~mm}$ ) such as those used by Burri et al. (2011b) to define a criterion for the onset of local sand entrainment. This, together with measurements of the local skin friction velocity time series, can then be used to improve predictions of sand erosion magnitudes.

\section{Summary and Conclusions}

Several distinctive differences between spatiotemporally-resolved surface shear-stress variations on the ground beneath live plant canopies and rigid cube arrays were found. Although the cubes provided a higher overall sheltering effect at the low and medium roughness densities, identical experiments with plants generated lower average and peak surface shear-stress partitions at the high roughness density. However, the plant canopies had lower absolute peak shear-stress values at all roughness densities than the rigid cube arrays. The plants streamline with the flow, resulting in a decreasing sheltering effect at higher wind velocities due to the narrower sheltered areas in their wakes. The patterns of surface shear stress on the ground beneath the plants and cubes suggest significant differences in erosion and deposition patterns in such canopies.

The peak near-ground turbulence intensities are about $40 \%$ higher in the sheltered area relative to the smooth floor case at all plant densities. In contrast, the peak turbulence intensities upwind and at the sides of the cubes are 120,70 and 50\% higher in the low, medium and high density cases relative to the smooth floor case. The turbulence intensity distributions support the hypothesis that horseshoe vortices are weakened or actually suppressed in the plant case.

The spatial patterns of the percentage of time when a certain threshold skin friction velocity is exceeded show compelling similarities to sand erosion and deposition patterns around plants found in a companion study (Burri et al. 2011b). We demonstrated that for our controlled experiments, net deposition (or no net erosion) is still possible even when the threshold value for erosion is locally exceeded $75 \%$ of the time, and that net erosion is possible even if the threshold is exceeded only $25 \%$ of the time.

Most importantly, all of our results demonstrate that rigid, non-porous roughness elements provide inadequate approximations of live plants. Results from many earlier studies using rigid roughness elements may thus have limited application in studies of the sheltering effect of live plants on sediment transport and soil erosion in natural environments. 
Acknowledgments We would like to thank the Swiss National Science Foundation (SNF) and the Vontobel foundation for financial support. The SLF workshop and GS technology assisted the development and the production of the measurement technique and the experiment set-up. Dr. Katrin Burri and Benjamin Eggert helped with the wind-tunnel experiments and provided many fruitful discussions.

\section{References}

Anderson RS, Sorensen M (1991) A review of recent progress in our understanding of aeolian sediment transport. Acta Mech 1:1-19

Bagnold R (1943) The physics of blown sand and desert dunes. Meghuen, London, 265 pp

Brown S, Nickling WG, Gillies JA (2008) A wind-tunnel examination of shear-stress partitioning for an assortment of surface roughness distributions. J Geophys Res 113:F02S06

Burri K (2011) Plants and mycorrhizal fungi on wind erosion control. Dissertation, ETH Zürich. doi:10.3929/ ethz-a-006570793

Burri K, Gromke C, Graf F (2011a) Mycorrhizal fungi protect the soil from wind erosion: a wind-tunnel study. Land Degrad Dev. doi:10.1002/ldr.1136

Burri K, Gromke C, Lehning M, Graf F (2011b) Aeolian sediment transport over vegetation canopies: a wind-tunnel study with live plants. Aeolian Res 3:205-213

Clifton A, Lehning M (2008) Improvement and validation of a snow saltation model using wind-tunnel measurements. Earth Surf Process Landf 33:2156-2173

Crawley DM, Nickling WG (2003) Drag partition for regularly-arrayed rough surfaces. Boundary-Layer Meteorol 107:445-468

Gillette DA, Stockton PH (1989) The effect of nonerodible particles on wind erosion of erodible surfaces. J Geophys Res 94:12885-12893

Gillies JA, Nickling WG, King J (2002) Drag coefficient and plant form response to wind speed in three plant species: Burning Bush (Euonymus alatus), Colorado Blue Spruce (Picea pungens glauca), and Fountain Grass (Pennisetum setaceum). J Geophys Res. doi:10.1029/2001JD001259

Gillies JA, Nickling WG, King J (2007) Shear-stress partitioning in large patches of roughness in the atmospheric inertial sublayer. Boundary-Layer Meteorol 122:367-396

Gromke C, Manes C, Walter B, Lehning M, Guala M (2011) Aerodynamic roughness length of fresh snow. Boundary-Layer Meteorol. doi:10.1007/s10546-011-9623-3

Irwin HPAH (1981) A simple omnidirectional sensor for wind-tunnel studies of pedestrian-level winds. J Wind Eng Ind Aerodyn 7:219-239

Kim DS, Cho GH, White BR (2000) A wind-tunnel study of atmospheric boundary-layer flow over vegetated surfaces to suppress PM $_{10}$ emission on Owens (dry) Lake. Boundary-Layer Meteorol 97:309-329

King J, Nickling WG, Gillies JA (2006) Aeolian shear-stress ratio measurements within mesquite-dominated landscapes of the Chihuahuan Desert, New Mexico, USA. Geomorphology 82:229-244

Lancaster N, Baas A (1998) Influence of vegetation cover on sand transport by wind: Field studies at Owens Lake, California. Earth Surf Proc Landf 23:69-82

Lyles L, Allison BE (1975) Wind erosion: Uniformly spacing nonerodible elements eliminates effects of wind direction variability. J Soil Water Conserv 30:225-226

Marshall JK (1971) Drag measurements in roughness arrays of varying density and distribution. Agric Meteorol 8:269-292

Morris HM (1955) Flow in rough conduits. Am Soc Civil Eng 120:373-398

Musick HB, Gillette DA (1990) Field evaluation of relationships between a vegetation structural parameter and sheltering against wind erosion. Land Deg Rehabil 2:87-94

Musick HB, Trujillo SM, Truman CR (1996) Wind-tunnel modelling of the influence of vegetation structure on saltation threshold. Earth Surf Proc Landf 21:589-605

Raupach MR (1992) Drag and drag partition on rough surfaces. Boundary-Layer Meteorol 60:375-395

Raupach MR, Gillette DA, Leys JF (1993) The effect of roughness elements on wind erosion threshold. Geophys Res 98:3023-3029

Schlichting H (1936) Experimental investigations of the problem of surface roughness. NASA Tech Memo 823 Washington

Shao Y (2008) Physics and modelling of wind erosion. Springer. ISBN:978-1-4020-8894-0, 452 pp

Sutton SLF, McKenna-Neumann C (2008) Variation in bed level shear-stress on surfaces sheltered by nonerodible roughness elements. J Geophys Res 113:F03016. doi:10.1029/2007JF000967

Valyrakis M, Diplas P, Dancey C, Greer K, Celik AO (2010) Role of instantaneous force magnitude and duration on particle entrainment. J Geophys Res. doi:10.1029/2008JF001247 
Walter B, Gromke C, Lehning M (2009) The SLF Boundary Layer Wind-tunnel-An Experimental Facility for Aerodynamical Investigations of Living Plants. In: 2nd international conference "Wind effects on trees", Freiburg, Germany, pp 31-37

Walter B, Gromke C, Leonard K, Clifton A, Lehning M (2011) Measurements of surface shear-stress distribution in live plant canopies. In: 13th international conference on wind engineering, Amsterdam, The Netherlands

Wolfe SA, Nickling WG (1996) Shear-stress partitioning in sparsely vegetated desert canopies. Earth Surf Proc Landf 21:607-619

Wooding RA, Bradley EF, Marshall JK (1973) Drag due to regular arrays of roughness elements of varying geometry. Boundary-Layer Meteorol 5:285-308

Wu H, Stathopoulos T. (1993) Further experiments on Irwin's wind sensor. J Wind Eng Ind Aerodyn 53: $441-452$

Wyatt VE, Nickling WG (1997) Drag and shear-stress partitioning in sparse desert creosote communities. Can J Earth Sci 34:1486-1498 